\title{
Una revisión de literatura sobre el uso de modelación y simulación computacional para la enseñanza de la física en la educación básica y media*
}

\author{
A literature review about computational modeling and simulation in physics education \\ in middle and high school levels
}

\author{
Sonia López ${ }^{\dagger 1}$, Eliane Angela Veit ${ }^{2}$, Ives Solano Araujo ${ }^{\ddagger 2}$ \\ ${ }^{1}$ Universidad de Antioquia, Facultad de Educación, Medellín, Colombia \\ ${ }^{2}$ Universidade Federal do Rio Grande do Sul, Instituto de Física, Porto Alegre, RS, Brasil
}

Recebido em 26 de dezembro de 2015. Aceito em 2 de fevereiro de 2016

\begin{abstract}
Este artículo presenta una revisión de la literatura que aborda los principales referentes epistemológicos, psico-pedagógicos y didácticos en las investigaciones sobre actividades de Modelación y Simulación Computacional (MSC) en la enseñanza de la física (en los niveles de educación básica y media). La búsqueda principal se llevó a cabo en las siguientes bases de datos: Science Direct, Scopus, Web of Science y Google Scholar considerando artículos publicados entre 2003-2013 y en busca de las siguientes palabras clave: "computational modeling"; "computational simulation"; physics teaching"; "research in physics teaching" (también las correspondientes palabras clave en español y portugués fueron usadas en la búsqueda). Después de la lectura de los artículos seleccionados, consideramos sólo las implementaciones de MSC que tenían como propósito contribuir al aprendizaje de conceptos físicos. Los resultados muestran que: i) las investigaciones se basan en las ideas epistemológicas contemporáneas, excepto un artículo con concepciones positivistas; ii) hay varias teorías cognitivistas siendo utilizadas (Piaget, Ausubel, Vergnaud,...), pero Vygostky es el más prominente; iii) estrategias que promueven las interacciones sociales son preponderantes en las actividades de resolución de problemas, simulaciones integradas con experimentos y modelación. De hecho, sólo dos (2) estudios entre los 21 encontrados sobre MSC en educación básica y media hacen uso de Modelación Computacional, lo que demuestra que es necesario un poco de esfuerzo para introducir e investigar este tipo de actividad en la enseñanza de la física. Sin embargo, identificamos una tendencia a aplicar un fundamento principalmente pedagógico y didáctico para la implementación de actividades de MSC en el aula de clase.

Palavras-chave: enseñanza de la física, Modelación y Simulación Computacional, concepción de ciencia, referentes pedagógicos y didácticos..
\end{abstract}

This paper presents a literature review covering the central epistemological, psychopedagogical and didactical framework on researches about Computational Modeling and Simulation (CMS) activities in physics education (Middle and High School levels). The main search was performed in the following databases: Science Direct, Scopus, Web of Science and Google Scholar considering articles published between 2003-2013 and looking for the following keywords: "computational modeling"; "computational simulation"; physics teaching"; "research in physics teaching". (Also the corresponding keywords in Spanish and Portuguese were used in the search). After reading the selected articles, we kept only those implementations of CMS aiming to contribute to the learning of physics concepts. The results show that: i) the investigations are based on contemporary epistemological ideas, except one paper with positivist conceptions; ii) there are various cognitivist theory being used (Piaget, Ausubel, Vergnaud,...), but Vygostky is the most prominent; iii) strategies that promote social interactions are preponderant in activities of problem solving, simulations integrated with experiments and modeling. In fact, only two (2) studies among the 21 found about CMS at Midle and Hich School make use of Computational Modeling, showing that it is necessary some effort to introduce and investigate these kind of activity in physics education. Nonetheless, we identify a tendency to apply a mostly pedagogical and didactical basis towards the implementation of CMS activities within the classroom.

Keywords: physics education, Computational Modeling and Simulation, conception of science, pedagogical and didactical basis.

\section{Introducción}

*En Brasil los niveles de educación básica y media equivalen al nivel fundamental.

${ }^{\dagger}$ Endereço de correspondência: sonia.lopez@udea.edu.co.

${ }^{\ddagger}$ Bolsista do CNPq - Brasil.
La incorporación de las Tecnologías de la Información y la Comunicación (TIC) en los procesos de enseñanza y aprendizaje se ha venido desarrollando de manera gradual durante varias décadas; y de 
modo particular, las tecnologías computacionales han tenido un gran impacto en el ámbito educativo, convirtiéndose en un importante campo de interés para la investigación en enseñanza de las ciencias $[1,2,3,4]$. En este sentido, Osborne y Hennessy [5] sugieren que las TIC crean el espacio para desarrollar el tipo de habilidades analíticas que exige la educación científica contemporánea.

Una mirada a publicaciones en enseñanza de las ciencias nos da una idea del importante uso que han adquirido las TIC en los últimos años. De tal manera que hoy en día es posible hablar de las TIC como una línea de investigación de la didáctica de las ciencias; entendiendo por línea de investigación "una sucesión continua e indefinida de estudios, reflexiones sistemáticas y creativas, indagaciones y discusiones alrededor de un problema, que realizan personas curiosas, enlazadas entre sí y organizadas en uno o varios equipos de trabajo para desarrollar actividades intelectuales y dinámicas, en medio de aciertos y desaciertos, logros, fracasos y éxitos, con el propósito común de construir y/o aumentar conocimientos sobre un determinado tópico" $[6, \mathrm{p}$. $1]$.

Desde esta perspectiva, en las últimas décadas varios autores [2, 3, 7 a 9] que han llevado a cabo importantes reflexiones sobre la investigación en la educación en ciencias, coinciden en abordar el tema de las TIC, considerándolas como un gran desafío y uno de los principales tópicos de investigación en educación en ciencias.

Justamente, el interés que se ha generado en los últimos años en esta línea de investigación de la didáctica de las ciencias se refleja en el creciente número de trabajos acerca del uso del computador en los procesos de enseñanza y aprendizaje, en mayor cantidad en el área de física y en menor medida en áreas como química y biología. Y es que el uso principalmente del computador como herramienta en los procesos de enseñanza y aprendizaje de las ciencias brinda varias posibilidades, como: tratamiento de cálculos, diseño de gráficas, instrumento de medición, instrumento de adquisición y procesamiento de datos, modelación y simulación de fenómenos científicos; siendo este último tipo de aplicación de las TIC, de acuerdo con Capuano [4], uno de los tipos de uso del computador más empleado en la enseñanza de las ciencias. Modos de uso que por sus características han tenido una mayor aplicabilidad en el área de física.
Tomando en cuenta estas consideraciones, se generó un proyecto de investigación cuyo propósito es caracterizar los presupuestos epistemológicos, psicopedagógicos y didácticos de los profesores de ciencias naturales (física, química y biología) de la educación básica y media que investigan sobre la implementación de actividades de Modelación y/o Simulación Computacional en su práctica docente. Y para este artículo en particular, en el que se presenta una revisión de literatura realizada simultáneamente al desarrollo del proyecto y enfocada a trabajos que hacen investigación sobre la implementación de actividades de MSC para la enseñanza de la física en la educación básica y media, se pretende hacer un acercamiento al tema a partir de preguntas relacionadas con:

\begin{abstract}
?Cuáles son las principales características (delimitación contextual, nivel educativo, temáticas abordadas, programas de MSC) de los trabajos de investigación sobre la implementación de actividades de MSC para la enseñanza de la física? y ¿cuáles son los principales referentes teóricos que fundamentan la investigación en la implementación de actividades de MSC para la enseñanza de la física?
\end{abstract}

Nuestro énfasis es exclusivamente en la Modelación y Simulación Computacional, asumiéndola como una modalidad de uso de las TIC altamente promisoria para favorecer el proceso de enseñanzaaprendizaje de la física, que posibilita el diseño e interacción con modelos conceptuales que, por lo menos en principio, puedan ser implementados en el computador y tengan fines didácticos.

A manera de antecedente, es importante mencionar que las revisiones de literatura más recientes que hemos conocido se han concentrado de modo general en el uso de TIC en la educación [5, 10 a 16]; otras, en el uso de TIC en la enseñanza de las ciencias [4, 17 a 19]. Y de manera más específica, localizamos una única revisión de literatura sobre el uso de TIC en la enseñanza de la física [20], que cuenta ya con más de diez años, justificando en buena medida la publicación de este artículo. En ninguna de ellas se aborda de manera particular el uso de la Modelación y/o Simulación computacional para la enseñanza de la física. 


\section{Acerca de la Modelación y Simulación Computacional en la Enseñanza de la Física}

La Modelación y la Simulación Computacional son dos términos utilizados en muchas ocasiones de manera indiscriminada, por lo que se hace necesario establecer claridad en relación con la manera en que cada uno es comprendido en el ámbito de este trabajo.

Al respecto, Lawrence [21] indica que existen diferentes tipos de actividades que pueden ser implementadas con el uso de herramientas computacionales, haciendo una escueta clasificación, de acuerdo con su uso, en actividades de animación, simulación y modelación. Desde su perspectiva, la animación permite la observación de una secuencia predeterminada, posibilitando (por decirlo de alguna manera) el sentir de los pensamientos de otros. Las simulaciones permiten al usuario hacer modificaciones en la entrada de valores y observar cómo varían los resultados, consiguiendo con esto una comprensión funcional de los pensamientos de otros. Por último, este autor concibe la modelación como una actividad que permite el cambio de reglas y de entrada de valores, observar la variación en los resultados obtenidos, facilitar una lectura de los pensamientos de otros, dándole la posibilidad de hacer modificaciones para expresar su propio pensamiento. Desde la perspectiva de este autor, es la habilidad para alterar las reglas lo que se entiende como modelación, siendo esta condición la que la hace diferente de actividades como la animación o la simulación.

En esta misma línea, Rogers [22] alude a la modelación y la simulación computacional, estableciendo una comparación entre los software utilizados para cada uno de estos propósitos. Aunque considera que ambos pueden ser usados para facilitar la investigación, la exploración de relaciones entre variables y la verificación de hipótesis, afirma que existe una obvia distinción en el diseño de la interface del usuario; ya que el software de modelación es usualmente de un diseño genérico, simbólico (por ejemplo: los software Stella, Excel y Modellus), mientras que las simulaciones usualmente ofrecen una interface gráfica personalizada a las necesidades del tema bajo consideración. Otra diferencia significativa que establece el autor, es que, aunque cada simulación emplea un modelo matemático como una "máquina" para realizar cálculos, el modelo usualmente no es explícito o accesible al usuario. En contraste con esto, el software de modelación permite el acceso a todas las definiciones matemáticas en el modelo a ser analizado y, si se desea, puede ser editado y modificado.

En este sentido, Araujo, Veit y Moreira [23], afirman que "estos dos tipos de actividades se distinguen por el acceso que el alumno tiene al modelo matemático o icónico subyacente a la implementación de la actividad". Es decir, desde la visión de estos autores, en una simulación computacional que representa un modelo físico, el alumno puede insertar valores iniciales para variables, alterar parámetros $\mathrm{y}$, de forma limitada, modificar las relaciones entre las variables; sin embargo, no tiene autonomía para modificar la estructura de la simulación (modelo matemático o icónico preespecificado); o sea, acceso a los elementos más básicos que la constituyen. La interacción del estudiante con la simulación tiene un carácter eminentemente exploratorio, mientras que en la modelación computacional, tiene acceso a los primitivos (estructura básica o fundamental) que constituyen el modelo computacional, pudiendo modificarlos, construirlos desde el principio y reconstruirlos conforme desee.

A partir de las consideraciones anteriores, se aclara que compartimos la visión de los últimos autores [23], y asumimos que la implementación de la modelación computacional en el aula representa tanto para profesores como estudiantes, una actividad altamente demandante que exige dominio de conceptos científicos y de los respectivos modelos matemáticos que constituyen la estructura del modelo computacional, así como el manejo (en muchos casos) de un determinado lenguaje de programación. Esta puede ser una de las razones por las cuales se encuentre en la revisión de literatura, un escaso número de trabajos en este campo, predominando la enseñanza de conceptos científicos a partir del uso de simulaciones computacionales.

\section{Modelación y Simulación Computacio- nal en la enseñanza de las ciencias a la luz de referentes epistemológicos, psico-pedagógicos y didácticos}

Aunque el interés del presente trabajo se limita a la enseñanza de la física como campo particular de la enseñanza de las ciencias, es importante remitirse a este contexto más amplio para comprender 
la implementación de actividades de Modelación y Simulación Computacional como una manera de recrear la actividad de modelación científica, entendida como un proceso de construcción, validación, uso y revisión de modelos científicos [24 a 29]. En este contexto, los modelos científicos son considerados representaciones idealizadas y simplificadas de la realidad, diseñados para describir, predecir y/o explicar una cierta situación problema relacionada con algún aspecto del mundo. Sin embargo, como lo menciona Brandão [30], el profesor de ciencias difícilmente reflexiona en el aula de clase sobre la naturaleza y el papel de los modelos científicos y del proceso de modelación en los contextos científico y educativo.

Investigaciones con y sobre profesores coinciden en señalar que una posible causa de esta dificultad se debe a que en muchas ocasiones sus concepciones epistemológicas son incompatibles con las concepciones actuales de la filosofía de la ciencia, siendo en muchos casos visiones inadecuadas, deformadas y descontextualizadas [31 a 35]. Y estas concepciones sobre ciencia en general están fuertemente relacionadas con las concepciones sobre modelos y modelado científico en el contexto de la física [29].

Por otro lado, las perspectivas psico-pedagógicas - que cumplen un papel relevante para quienes se interesan en los procesos de aprendizaje y construcción de conocimiento por parte de los estudiantes - se encuentran regularmente consignadas en los proyectos educativos institucionales y planes de área que orientan al profesor de educación básica y media en su quehacer docente; no obstante, difícilmente existe un real conocimiento de dichos referentes y por tanto, éstos no trascienden hacia su práctica pedagógica. Y cuando en el aula de clase son implementadas algunas estrategias de enseñanza, como por ejemplo, la Modelación y Simulación Computacional, no necesariamente estos fundamentos teóricos entran en juego para soportar y direccionar el desarrollo de tales actividades.

En lo que al saber didáctico se refiere, es necesario que los profesores de ciencias replanteen el uso de las tecnologías computacionales - particularmente de la MSC - para la enseñanza de las ciencias, en términos del propósito didáctico con el que éstas son introducidas en el ambiente del aula; dado que el principal objetivo de la utilización de cualquier estrategia para la enseñanza de las ciencias es que posibilite el aprendizaje de conceptos científicos. Al respecto, algunos autores [36, 37] consideran que una utilización de software que se limite a poner al alumno frente a la simulación, sin adecuada interacción con el docente y sin el control de ciertas variables didácticas por parte de éste, podría conducir a resultados opuestos a los esperados, como aprendizajes puramente mecánicos. En estos términos, toman relevancia no sólo la perspectiva didáctica en la que se inscribe el maestro, sino además la coherencia que tenga con las estrategias de enseñanza, aprendizaje y evaluación que elige para llevarlas al aula.

En este orden de consideraciones, es importante no desconocer el aporte de las tecnologías computacionales en la facilitación de procesos propios de la ciencia, como la modelación y la simulación de fenómenos científicos; pero más importante aún, es asumir una actitud crítica frente a la implementación de las TIC en los procesos de enseñanza y aprendizaje de las ciencias, lo que implica un proceso de reflexión por parte de los profesores de ciencias en relación con aspectos de corte epistemológico, psico-pedagógico y didáctico.

\section{Metodología}

La presente revisión de literatura se enmarca en una investigación documental mayor que busca conocer el estado actual - estado del arte- de la investigación en el campo de la Modelación y Simulación Computacional en la enseñanza de las ciencias naturales; y se presentan aquí de manera particular, los resultados para el área de física. Para su desarrollo, se contemplan algunos elementos metodológicos planteados por Hoyos [38], como los núcleos temáticos, considerados los subtemas que delimitan el campo de conocimiento; es decir, aquellos asuntos que ayudan a acotar el objeto de estudio y a identificar con mayor facilidad las fuentes de información. Otro elemento metodológico lo constituyen las unidades de análisis, que hacen referencia a un texto individual (cualquiera que sea su carácter: libro, ensayo, tesis, artículo, etc.) seleccionado para revisión y análisis. Además, a los aspectos que destacan elementos de relevancia a señalar o a distinguir en una unidad de análisis, se les denomina factores.

Atendiendo a estas recomendaciones metodológicas fueron definidos tres núcleos temáticos denominados: referentes epistemológicos del uso de MSC en enseñanza de la física, referentes psico-pedagógicos del uso de MSC en enseñanza de la física y refe- 
rentes didácticos del uso de MSC en enseñanza de la física; núcleos a la luz de los cuales se valora la totalidad de las unidades de análisis. Y en concordancia con el propósito de la investigación, los factores considerados a partir de cada unidad de análisis se refieren a aspectos como la delimitación contextual, el nivel educativo en el que se implementa la propuesta, las temáticas de física abordadas, así como los principales programas de modelación y/o simulación computacional utilizados; elementos que toman relevancia en el proceso de contextualización, análisis e interpretación de cada unidad de análisis.

Asimismo, se recurre al análisis de contenido como procedimiento de recolección y análisis de la información; entendiendo este como el conjunto de procedimientos interpretativos de productos comunicativos (mensajes, textos o discursos) que provienen de procesos singulares de comunicación previamente registrados, que tienen por objeto elaborar y procesar datos relevantes sobre las condiciones mismas en que se han producido aquellos textos, o sobre las condiciones que puedan darse para su empleo posterior [39].

En una primera etapa, la búsqueda se realizó en bases de datos y motores de búsqueda como Science Direct, Scopus, Web of Science y Google Scholar, haciendo uso de diferentes palabras clave en los idiomas español (modelación computacional, simulación computacional, enseñanza de física, investigación en enseñanza de la física), inglés (computational modeling, computational simulation, physics teaching, research in physics teaching) y portugués (modelagem computacional, simulação computacional, ensino de física, pesquisa em ensino de física).

Posteriormente, se realizó una búsqueda manual en revistas incluidas en las bases de datos y en otras que consideramos relevantes en el área; para un total de 34 revistas (American Journal of Physics, Physics Education, Philosophy of Science, The Physics Teacher, School Science and Mathematics, American Educational Research, Research in Science Education, Teachers and Teaching: theory and practice, Journal of Science Education and Technology, Journal of Computer Assisted Learning, International Journal of Science Education, Latin American Journal of Physics Education, Journal of Research in Science Teaching, Journal of Science Teacher Education, Science Education, Science 83 Education, Computers \& Education, Revista Ciência \& Edu- cação, Revista Enseñanza de las Ciencias, Revista electrónica de Enseñanza de las Ciencias, Revista Iberoamericana de Tecnología en Educación y Educación en Tecnología, Revista Latinoamericana de Enseñanza de la Física, Revista Eureka sobre Enseñanza y Divulgación de las Ciencias, Revista Mexicana de investigación Educativa, Revista de Educación en Ciencias, Revista Chilena de Educación Científica, Tecné, Episteme y Didaxis: TED, Revista Investigações em Ensino de Ciências, Revista Brasileira de Pesquisa em Educação em Ciências, Caderno Brasileiro de Ensino de Física, Revista Brasileira de Ensino de Física, Revista de investigación, Revista de enseñanza de la física, Experiencias em ensino de ciencias); en las que fueron inicialmente identificados ciento cincuenta y cuatro (154) artículos.

No obstante, un importante número de estos trabajos se relacionaba de manera tangencial con el objeto de estudio de la revisión de literatura que aquí presentamos, por lo que no todos fueron considerados relevantes para el análisis, dado que no guardaban una profunda correspondencia con los propósitos de este estudio. Entre estos se incluyen los relativos a: reflexiones de carácter general sobre la MSC en la enseñanza de las ciencias, reflexiones sobre el uso de TIC en la Educación, modelación (no computacional) en ciencias o en otras áreas, reflexiones generales sobre el uso de simulaciones computacionales en ciencias y revisiones de literatura sobre el tema.

Del mismo modo, no fueron considerados para esta revisión trabajos relacionados con la MSC que se limitaban a la descripción del uso de software, mostrando ejercicios y actividades posibles de realizar, o a la descripción de las ventajas de un programa determinado, sin detallar la implementación en el aula de clase. Aunque que se encontró un gran número de estos trabajos, ellos fueron descartados para el análisis en coherencia con los núcleos temáticos definidos para la selección de las unidades de análisis; donde se tuvieron en consideración exclusivamente aquellos trabajos que describen la implementación efectiva de actividades de Modelación y/o Simulación Computacional en la enseñanza de la física, que buscan contribuir al aprendizaje de conceptos físicos. $\mathrm{Al}$ tener en consideración este criterio el número de trabajos se reduce tan solo a sesenta y cuatro (64).

Finalmente, al centrar el interés de este estudio en los trabajos que describen la implementación 
efectiva de actividades de MSC en la enseñanza de la física concretamente en los niveles de educación básica y media, el número de trabajos que cumple con este criterio y que se constituye en unidades de análisis para esta investigación, se reduce a veintiuno (21).

\section{Resultados y Discusión}

Como ya fue mencionado, este artículo hace parte de un proyecto de investigación donde uno de sus componentes es una revisión de literatura mucho más amplia en la que se aborda el uso de la Modelación y Simulación Computacional para la enseñanza de la física, la química y la biología en la educación básica y media. Los resultados de esta revisión nos muestran que el uso de MSC en la enseñanza de las ciencias se ha concentrado más en unas disciplinas que en otras, siendo la física el área con mayor producción en el campo, en comparación con el número de artículos encontrados para las áreas de biología y química. Asimismo, la implementación de actividades de MSC se concentra más ampliamente en el nivel universitario, siendo muy reducido el número de trabajos de investigación para los niveles de educación básica y media.

Aunque cada vez existen más apuestas por incorporar herramientas TIC para abordar principalmente la enseñanza de algunos conceptos físicos que suelen tornarse un tanto complejos para los estudiantes, el número de trabajos se reduce significativamente cuando el interés se centra de manera específica en aquellos que hacen uso exclusivamente de actividades Modelación y/o Simulación Computacional; aun considerando la simulación como el tipo de aplicación de las TIC mayoritariamente empleado en la enseñanza de las ciencias [4].

Para el período de revisión 2003-2013, en las fuentes de información exploradas, se encontraron sesenta y cuatro (64) artículos que describen la implementación efectiva de actividades de MSC en la enseñanza de la física que buscan contribuir al aprendizaje de conceptos físicos; incluyendo aquellos trabajos que implementan estas actividades en el nivel universitario. Las revistas en las que se encuentran publicados estos trabajos y el número de ellos por revista de acuerdo con el nivel educativo, se muestran en la tabla 1.

La mayoría de trabajos referentes al uso de MSC en la enseñanza de la física fue encontrada en revis- tas del campo de la enseñanza de las ciencias (30 artículos), seguido por los trabajos publicados en revistas del ámbito de la enseñanza de la física (26 artículos); mientras que en revistas del ámbito de la investigación educativa y/o tecnología, se encontró únicamente un artículo por revista, con excepción de la revista Computers \& Education donde fueron encontrados cuatro (4) artículos, para un total de ocho (8) artículos.

No obstante, teniendo en consideración el interés de este estudio en los trabajos que describen la implementación efectiva de actividades de MSC en la enseñanza de la física en los niveles de educación básica y media, el número de trabajos que cumplen con este criterio y que se constituyen en unidades de análisis para esta investigación, se reduce a 21 (6 trabajos para la educación básica y 15 para la educación media), siendo los otros 43 trabajos implementados en el nivel superior o universitario.

En este orden de consideraciones, el análisis se concentra exclusivamente en estos 21 trabajos, publicados principalmente en revistas como: Journal of Science Education and Technology, Journal of Science Education, Latin American Journal of Physics Education Revista Brasileira de Ensino de Física y Experiências em Ensino de Ciências. Aquí es importante resaltar que, igual que en el caso en el que se incluían los trabajos a nivel universitario, el mayor número de trabajos de MSC en la enseñanza de la física en el nivel básico y medio, también es reportado en revistas del ámbito de investigación en la enseñanza de las ciencias (14 artículos).

La enseñanza de la física se ha convertido en un tema de interés tanto a nivel latinoamericano como en el ámbito mundial, ya que muchas teorías y modelos propios de esta disciplina tienen un nivel muy alto de abstracción y por ende, tanto en los niveles básicos como superiores de educación, se requiere de mayores esfuerzos a la hora de abordarse. Posiblemente como consecuencia de esto, la revisión de literatura aquí presentada arroja un importante número de trabajos que se enfocan en mostrar cómo los software para la Modelación y Simulación Computacional se han convertido en herramientas de gran ayuda para la enseñanza de conceptos físicos.

Una revisión de estos trabajos a la luz de los factores de análisis previamente definidos, nos aporta entre otras cosas, información relacionada con su procedencia. De las 21 unidades de análisis, se destaca que un número significativo de estas corresponde 
Tabla 1: Número de artículos que implementan MSC en la enseñanza de la física, en los diferentes niveles educativos (década 2003-2013).

\begin{tabular}{|c|c|c|c|c|}
\hline Ámbito de la Revista & Nombre de la Revista & $\begin{array}{l}\text { Educación bási- } \\
\text { ca (primaria y } \\
\text { secundaria) }\end{array}$ & $\begin{array}{l}\text { Educación } \\
\text { media }\end{array}$ & $\begin{array}{l}\text { Educación su- } \\
\text { perior }\end{array}$ \\
\hline \multirow{5}{*}{$\begin{array}{l}\text { Investigación en } \\
\text { educación y/o } \\
\text { tecnología }\end{array}$} & Revista de Investigación & \multirow{5}{*}{1} & 1 & \\
\hline & Computers \& Education & & 1 & 3 \\
\hline & Journal of Computer Assisted Learning & & & \\
\hline & Revista Mexicana de investigación Educativa & & & 1 \\
\hline & $\begin{array}{l}\text { Revista Iberoamericana de Tecnología en Edu- } \\
\text { cación y Educación en Tecnología }\end{array}$ & & & 1 \\
\hline \multirow{12}{*}{$\begin{array}{l}\text { Investigación en } \\
\text { enseñanza de las } \\
\text { ciencias }\end{array}$} & Journal of Science Education & 1 & 1 & 1 \\
\hline & Revista Chilena de Educación Científica & & 1 & \\
\hline & Tecné, Episteme y Didaxis: TED & 1 & & 1 \\
\hline & Journal of Science Education and Technology & 2 & 3 & \\
\hline & Experiências em Ensino de Ciências & \multirow{8}{*}{1} & 3 & \\
\hline & International Journal of Science Education & & 1 & 1 \\
\hline & Journal of Research in Science Teaching & & & 3 \\
\hline & Revista Enseñanza de las Ciencias & & & 2 \\
\hline & Revista electrónica de enseñanza de las ciencias & & & 5 \\
\hline & $\begin{array}{l}\text { Revista Eureka sobre Enseñanza y Divulgación } \\
\text { de las Ciencias }\end{array}$ & & & 1 \\
\hline & Ciência \& Educação & & & 1 \\
\hline & Investigações em Ensino de Ciências & & & 1 \\
\hline \multirow{5}{*}{$\begin{array}{l}\text { Investigación en } \\
\text { enseñanza de la } \\
\text { física }\end{array}$} & Revista Brasileira de Ensino de Física & & 2 & 6 \\
\hline & Latin American Journal of Physics Education & & 2 & 6 \\
\hline & American Journal of Physics & & & 3 \\
\hline & Physics Education & & & 3 \\
\hline & Caderno brasileiro de ensino de física & & & 4 \\
\hline Total & & 6 & 15 & 43 \\
\hline
\end{tabular}

Tabla 2: Número de artículos por país.

\begin{tabular}{ll}
\hline País & Número de artículos \\
\hline España & 1 \\
Estados Unidos de América & 1 \\
India & 1 \\
Finlandia & 2 \\
Brasil & 5 \\
Holanda & 2 \\
China & 1 \\
Argentina & 2 \\
Israel & 2 \\
Grecia & 3 \\
Chile & 1 \\
\hline
\end{tabular}

a países latinoamericanos, siendo Brasil el país con más aportes (5 artículos), seguido por Argentina (2 artículos); inclusive superando a Estados Unidos de América, donde se identifica solo una publicación en este campo. En la tabla 2 se muestra el número de artículos encontrados por país.

Otro factor o elemento relevante de las unidades de análisis, nos indica que la MSC se ha implementado principalmente para abordar campos conceptuales de la física, tales como: mecánica clásica [40 a 45], electricidad y magnetismo [46 a 54], óptica [55 a 58] y mecánica cuántica [59, 60]; lo que se constituye en un panorama bastante alentador, si se tiene en consideración que hasta hace poco la MSC era usada prioritariamente para la enseñanza de conceptos referentes al campo de la mecánica clásica; lo cual parece estar siendo superado con los trabajos en otros campos que se caracterizan por una mayor complejidad.

Lo anterior, puede ser producto de la diversidad de software con que se cuenta actualmente para la enseñanza de conceptos físicos y posiblemente con la formación de profesores de física, cada vez más conscientes de la necesidad de incluir herramientas tecnológicas dentro de la gama de estrategias didácticas necesarias para favorecer el aprendizaje de la física. No obstante, sorprende encontrar que en el ámbito de la mecánica cuántica, se cuente solamente con dos trabajos que abordan la enseñanza de este campo de conocimiento con el uso de modelos y simulaciones en el nivel medio; más aún, considerando el alto grado de abstracción de los conceptos propios de este campo, y por ende, la posibilidad 
que tiene el profesor de apoyarse en representaciones que favorezcan su comprensión, aprovechando la potencialidad que brindan los modelos y simulaciones computacionales.

Un elemento que se pone de manifiesto en los trabajos analizados es que la gran mayoría de éstos (19) hacen uso de actividades de simulación computacional; y tan sólo dos trabajos [41, 45] se refieren a la modelación. El contraste de este hallazgo con lo que se encuentra comúnmente en la literatura no genera sorpresa alguna, pero sí se constituye en una oportunidad para reflexionar en torno al escaso uso de actividades de modelación computacional en comparación con las actividades de simulación; lo que se convierte sin duda en un indicador de la complejidad que representa, tanto para profesores como estudiantes, la implementación de actividades de modelación computacional en el aula. Pues, como ya fue mencionado, estas requieren necesariamente del dominio no solo de conceptos científicos y los respectivos modelos matemáticos que constituyen la estructura del modelo computacional, sino también (en muchos casos), de un determinado lenguaje de programación.

En el caso de los dos trabajos que hacen uso de actividades de modelación computacional, ambos utilizan el software Modellus [41, 45], un software gratuito, que posee una interface gráfica intuitiva, facilitando la interacción de los estudiantes con modelos en tiempo real y el análisis de múltiples representaciones de esos modelos. Este software es apropiado para el desarrollo de modelos computacionales referentes al campo de la dinámica Newtoniana; siendo efectivamente utilizado en estos dos trabajos para abordar el campo de la mecánica clásica. No obstante, este programa puede ser perfectamente utilizado para construir modelos computacionales en otros campos de la física; y de hecho, es utilizado en dos de los trabajos para abordar el estudio de la electricidad y el magnetismo [50] y la mecánica cuántica [60]; aunque no como actividades de modelación, sino de simulación computacional.

En esta misma línea de las actividades de simulación computacional, fueron empleados diversos software, aplicaciones y/o lenguajes de programación; y si bien, el propósito de este trabajo no se centra en describir los programas usados en actividades de MSC, se hace una breve presentación de los mismos, para comprender mínimamente algunas razones para su elección. Uno de ellos es el Visual
Basic [46], un lenguaje de programación para $M i$ crosoft, diseñado para la creación de aplicaciones de manera productiva, y orientado a objetos. Asimismo, es utilizado el Modelscreator [40], el cual constituye un entorno de aprendizaje abierto destinado a facilitar la creación y el diseño de modelos, la exploración de su comportamiento, su mejora y, posiblemente, la validación de sus límites.

Como un programa especialmente apropiado para abordar temas de astronomía, se encuentra el Planetarium [43], un software que permite al usuario simular el comportamiento del cielo en el día y en la noche, aportando un panorama 3D de la Vía Láctea. Y para el estudio de esta misma temática nos encontramos con la aplicación Virtual Solar System [42], que no se constituye en un software como tal, sino más bien en una simulación dinámica, basada en imágenes reales de la NASA de más de 90 objetos celestes en el sistema solar tales como planetas, cometas, principales estrellas, entre otros.

Encontramos también el uso del software Co-Lab [48], el cual proporciona un entorno de aprendizaje en línea con un lenguaje de modelado, así como tablas y gráficos para mostrar los resultados de la ejecución del modelo; que permite a los estudiantes experimentar con simulaciones y laboratorios remotos.

El software Cocodrile physics [49], es un softwa$r e$ educativo con potencialidades para construir simulaciones, gráficas y experimentos de física, especialmente en temáticas como electricidad, ondas, dinámica y óptica; y para abordar este último campo de conocimiento, se presenta el software Toolbok [55], en el que se crea una pieza denominada Reflection and Diffusion, una herramienta utilizada para crear aplicaciones multimedia, que permite cambiar el flujo de la información según las necesidades del usuario, relacionar imágenes o palabras, mostrar videos, entre otras.

En dos de los trabajos [51, 53] se hace uso de una simulación online denominada Electricity Exploration Tool, una aplicación de Abode Flash que permite realizar actividades como: videoconferencias, juegos en red, edición y reproducción de vídeo, animaciones 3D y chat. Un trabajo más hace uso de SimQuest [52] que es un entorno para la creación de ambientes de aprendizaje, cuyas aplicaciones hacen uso de simulaciones en áreas disciplinares como la física, la química o la biología. 
Encontramos así, el uso de diferente software y aplicaciones empleadas para incorporar actividades de simulación computacional en la enseñanza de conceptos físicos. No obstante, la mayor tendencia se concentra en las simulaciones que hacen uso del lenguaje Java [54, 56 a 58], un lenguaje de programación orientado a objetos y a partir del cual se construye un número importante de applets, o pequeñas aplicaciones que se ejecutan en navegador web.

De los trabajos analizados encontramos además que uno de ellos [59] hace referencia al uso de simulaciones libres sin entrar a especificar el tipo de aplicación; y del mismo modo, otros dos trabajos [44, 47] hacen referencia al uso de simulaciones, pero no se especifica el software o lenguaje de programación utilizado para su construcción.

El predominante uso de actividades de simulación computacional en comparación con las actividades de modelación es sin duda un indicador de la complejidad que señalábamos en párrafos anteriores, refiriéndonos a las dificultades que estas últimas generan en términos de del dominio requerido por parte de estudiantes y profesores, no solo de conceptos científicos y los respectivos modelos matemáticos que constituyen la estructura del modelo computacional, sino también (en muchos casos), de un determinado lenguaje de programación, que propicie la construcción de dicho modelo.

En la tabla 3 se muestran los principales factores que permiten realizar una caracterización general de los trabajos revisados.

En una disciplina como la física que se ha distinguido por ser el área de las ciencias naturales mayormente atendida por el uso de tecnologías computacionales en sus procesos de enseñanza, se considera que 21 trabajos en la modalidad de MSC es un número realmente bajo; no obstante, estos pueden aportarnos importantes indicios en relación con los referentes psico-pedagógicos en que se sustentan, las perspectivas y/o estrategias didácticas que orientan la implementación de la MSC; y las concepciones de ciencia que se vislumbran en la manera como estas actividades son implementadas en el aula de clase.

\subsection{Principales referentes epistemológicos para la MSC en la enseñanza de la física}

Es importante aclarar que este núcleo temático tiene como propósito identificar la concepción de la naturaleza de la ciencia que se devela en los trabajos revisados; teniendo en consideración, que dicha concepción normalmente aparece implícita. Esto implicó realizar una lectura crítica de cada uno de los trabajos y llevar a cabo un proceso de interpretación por parte de los autores, enfocándonos principalmente en asuntos como la concepción de modelo que se vislumbra en cada estudio, la concepción del proceso de construcción de conocimiento y de aprendizaje, entre otros.

De esta manera, encontramos que en la gran mayoría de los trabajos [ 40 a 47,49 a 53,55 a 60 ] se deja entrever la ciencia como una construcción social y colectiva, que considera a los estudiantes sujetos de conocimiento y se aleja de posturas dogmáticas en relación con el proceso de construcción del conocimiento. Muchos de ellos critican explícitamente el método tradicional de la actividad experimental en ciencias, donde se da prioridad a la recolección de datos y a la formulación de hipótesis, dejando en un segundo plano el análisis de los resultados y el desconocimiento del papel que la teoría juega en dicho análisis; y proponiendo una actividad experimental en la que los estudiantes adquieran previamente un dominio teórico que logren integrar con los procesos de obtención y análisis de datos.

En otro de los trabajos [48], se vislumbra una concepción con una tendencia más positivista del conocimiento científico; esto, si se tiene en cuenta que fue llevada a cabo una intervención que daba mayor protagonismo al manejo y análisis de los datos obtenidos y menos a la interacción entre los estudiantes para propiciar la construcción y comprensión de significados; de tal manera, que las actividades fueron realizadas de modo individual, reforzando así una concepción individualista y elitista de la ciencia [61]. Asimismo, este trabajo se centró en una metodología de investigación eminentemente cuantitativa que buscaba comparar el desempeño de un grupo control y un grupo experimental, desestimando los resultados de tipo cualitativo que pudieran ser relevantes para la comprensión del estudio. Siendo esto último similar a lo que sucede con otro de los trabajos [54], pero que no por ello podríamos enmarcarlo necesariamente en una concepción positivista de la ciencia.

\subsection{Principales referentes psico-pedagógi- cos para la MSC en enseñanza de la físi- ca}

La tarea de enseñar implica una relación directa con el aprendizaje, concepto que a su vez conlleva diversas tensiones entre lo psicológico y lo pedagógi- 


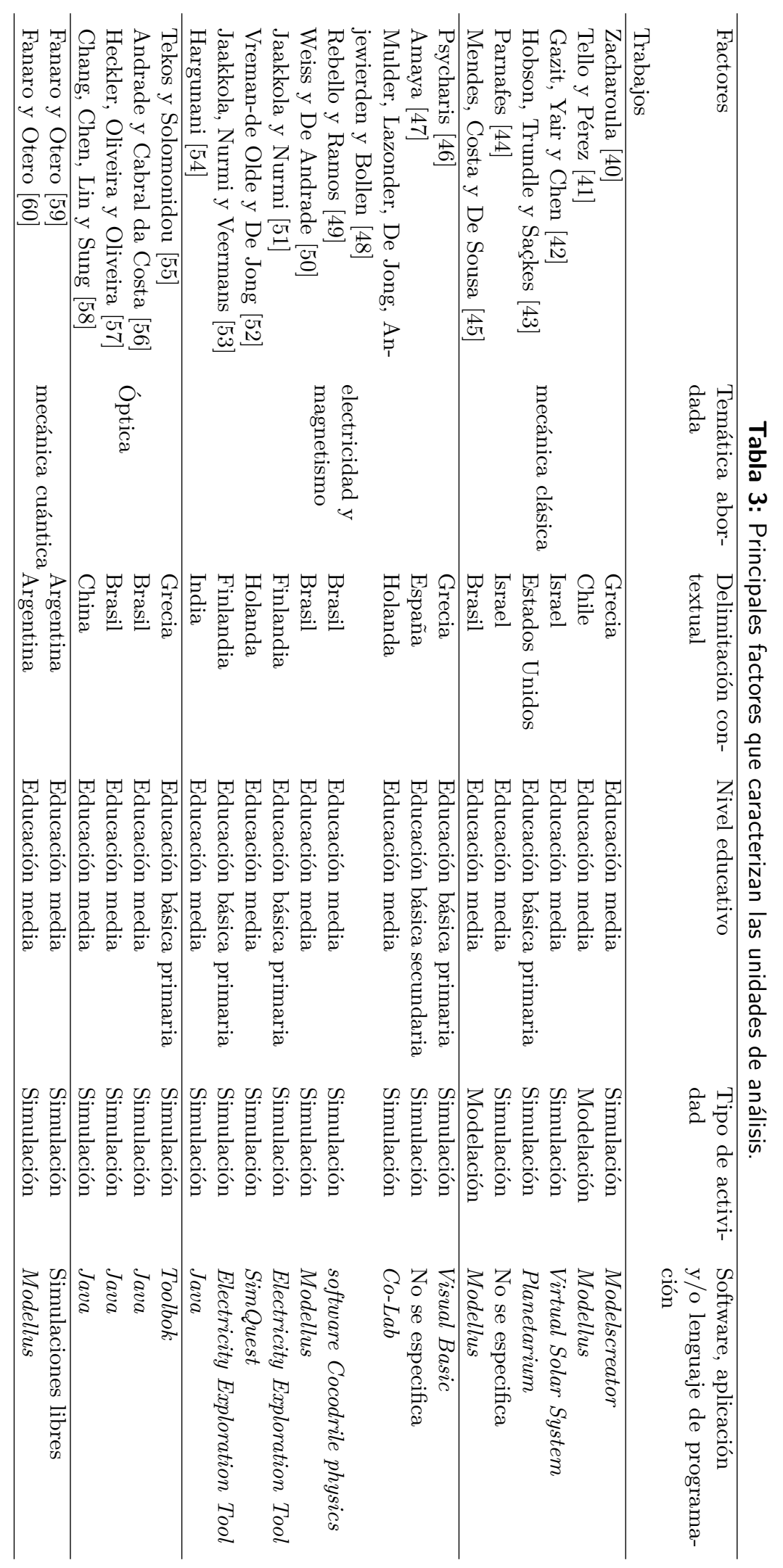


co, como vías posibles para entender lo que sucede en el sujeto que aprende. Dentro de un enfoque constructivista-cognitivista del aprendizaje, se considera que el estudiante construye de forma activa su propio conocimiento en el contexto social en el que se desenvuelve y a partir de su conocimiento anterior [62]. O como diría Ausubel [63], el aprendizaje se constituye en una tarea de construcción esencialmente idiosincrásica en cada individuo, que depende en forma exclusiva de lo que el alumno ya sabe.

No obstante, además del enfoque constructivistacognitivista del aprendizaje, en el que se enmarca la mayoría de propuestas de enseñanza actuales, existen otros enfoques teóricos como el conductismo, que sigue presente aún en muchas prácticas educativas, y el humanismo, que tiene como propósito fundamental profundizar en el mundo subjetivo del hombre para desarrollar lo mejor de él, lograr su crecimiento humano y su autorrealización.

De estos tres enfoques se derivan diversas teorías de aprendizaje, que se constituyen en construcciones humanas que buscan interpretar sistemáticamente aquello que llamamos aprendizaje. Estas teorías pretenden describir y explicar cómo se produce el aprendizaje; es decir, cómo aprenden aquí y ahora los seres humanos.

En lo que se refiere al enfoque constructivistacognitivista predominante en la actualidad, puede verse este como un enfoque filosófico que se ocupa de cómo el individuo conoce a partir de la construcción de su propia estructura cognitiva, pues los principios o postulados de este enfoque coinciden en señalar que el desarrollo y el aprendizaje humanos son básicamente el resultado de un proceso de construcción. De este modo, el constructivismo-cognitivismo es una concepción educativa y psicológica fundamentada en la importancia crucial que tiene para los individuos el construir significados. Por su parte, la construcción del conocimiento se concibe como un proceso en el cual interactúan los conocimientos adquiridos (conceptos previos o preconcepciones) y la información nueva que procede normalmente del material de aprendizaje. A partir de estas dos fuentes de información el sujeto construye nuevos conocimientos.

En el análisis de los trabajos que implementan actividades de MSC para la enseñanza de conceptos físicos, encontramos que dos de ellos [40, 54] no hacen explícitos sus referentes teóricos relacionados con el aprendizaje; y todos los demás, enmarcan sus reflexiones en un enfoque constructivista-cognitivista, en buena coherencia con la perspectiva epistemológica que se devela en la mayoría de ellos; aunque algunos $[52,58]$ no especifican un referente teórico concreto.

Dentro de este enfoque particular, encontramos varias tendencias en relación con diversas teorías de aprendizaje y autores que hacen sus aportes al mismo. Así, un importante número de trabajos se sustenta en la perspectiva sociocultural de Vygotsky $[44,47,49,55,57]$ desde la cual, el desarrollo cognitivo no puede entenderse sin referencia al contexto social, histórico y cultural en el cual ocurre.

Asimismo, un número significativo de trabajos se fundamenta en el Cambio Conceptual [42 a 44, 50, $51,53,55]$, pero asumiendo éste desde una perspectiva del desarrollo, enriquecimiento, comprensión y evolución conceptual [64 a 66]. En este orden de tendencias, encontramos algunos trabajos $[41,45$, 50] apoyados en la Teoría del Aprendizaje Significativo [63]; una teoría constructivista-cognitivista que pone de relieve la importancia y el papel de los conocimientos previos, en la adquisición de nuevos aprendizajes; y en esa misma línea, hallamos un trabajo [49], que además de sustentarse en la perspectiva sociocultural de Vygotsky, retoma elementos de la visión ausubeliana, pensados para favorecer el aprendizaje significativo en el aula de clase; es decir, la Teoría del Aprendizaje Significativo Crítico, formulada por Moreira [67]; un referente teórico que a partir de sus principios o estrategias facilitadoras del aprendizaje significativo crítico susceptibles de ser implementadas en el aula, aporta las condiciones para que el estudiante construya su conocimiento desde una perspectiva crítica y reflexiva.

Del mismo modo, otros trabajos encuentran un sustento psico-pedagógico en perspectivas teóricas como: la Teoría de los campos conceptuales de Vergnaud $[59,60]$, en la que el autor retoma elementos de Piaget y Vygotsky; la Teoría de la enseñanza de Bruner [46]; y la teoría de los modelos mentales de Johnson-Laird [56].

\subsection{Perspectivas $\mathrm{y} / \mathrm{o}$ estrategias didácticas que orientan la implementación de la MSC en la enseñanza de la física}

En lo que se refiere a la perspectiva y/o estrategia didáctica, para este núcleo se tienen en cuenta entre otros elementos: los diferentes enfoques y/o estrategias didácticas desde las cuales se orienta el uso de MSC computacional para la enseñanza de conceptos físicos, los medios propuestos para que el estudiante interactúe con la aplicación, así como la 
implementación del trabajo individual o colectivo; de tal manera que se pone de relieve una predominancia del trabajo grupal que propicie discusiones e interacción social entre el grupo, identificándose un número significativo de trabajos que hace uso de esta estrategia [ 42 a $45,47,50,51,54$ a 57,59$]$; dos trabajos más [48, 52] proponen la implementación de simulaciones computacionales desde un ejercicio individual; y los demás no hacen claridad en relación con el uso de actividades individuales o colectivas.

En lo relativo a las estrategias didácticas desde las cuales es acompañada la actividad de modelación y/o simulación computacional en el aula de clase, una de las más utilizadas es la resolución de problemas [49, 52, 58, 59], al igual que el método POE (Predecir, Observar, Explicar), estrategia utilizada por Tao y Gunstone [68] para la exploración de modelos y/o simulaciones computacionales; y que es explícitamente utilizado en dos de los trabajos [45, $50]$, e implícitamente se hace uso de éste en otros dos $[58,60]$.

La estrategia de generación y formulación de preguntas por parte del profesor y de los estudiantes, también ha ganado bastante terreno en el aula de clase. Al respecto encontramos tres trabajos que orientan el uso de simulaciones computacionales desde esta perspectiva $[42,52,59]$. Asimismo, un número significativo de trabajos $[45,49,51,53]$ hace uso de las actividades de simulación computacional en integración con actividades prácticas de laboratorio, estableciendo en muchas ocasiones una comparación entre los resultados obtenidos. En menor medida, encontramos el uso de mapas conceptuales y crucigramas [46], así como el uso de una secuencia didáctica [60] constituida por situaciones que dan sentido a los conceptos, desde la perspectiva de la Teoría de los Campos Conceptuales.

Más allá de las estrategias didácticas más comúnmente utilizadas para acompañar las actividades de MSC, nos encontramos con referentes didácticos de mayor complejidad, pero altamente valiosos y apropiados para la orientación de estas actividades en el aula de clase. Desde esta perspectiva, tenemos que uno de los trabajos [59] que hace uso de las simulaciones computacionales para abordar la enseñanza de la mecánica cuántica, asume como referente didáctico la transposición didáctica desde la perspectiva de Chevallard [69], fundamentándose en la idea de que la mecánica cuántica de los físicos profesionales resultará inevitablemente transformada cuando se intente enseñarla en la escuela.
En este orden de consideraciones, otro de los trabajos aquí revisados [49] hace uso de las unidades de aprendizaje, considerándolas como una posibilidad para la investigación en las clases de física. Desde esta perspectiva, la unidad de aprendizaje es definida como un modo de organización curricular, que busca superar la planeación secuencial y lineal de conceptos, valora los conocimientos de los alumnos con miras a su reconstrucción y complejidad; con el propósito de promover aprendizajes significativos. Asimismo, las unidades de aprendizaje intentan favorecer la capacidad de pensar, de solucionar problemas y desarrollar la autonomía y la autoría [70].

Como otro referente didáctico altamente pertinente y que se constituye en una estrategia para apoyar las actividades de simulación computacional en la enseñanza de la física, en uno de los trabajos revisados [41] es propuesto el modelamiento mental desde la perspectiva de Hestenes [71, 72]; desde la cual se plantea la actividad de modelamiento como un ciclo constituido por dos etapas: el desarrollo del modelo (que contempla la descripción, formulación, ramificación y validación del modelo) y el uso del modelo (referente a la aplicación del mismo). Desde la perspectiva de este autor [71], cada paso es esencial para modelar y la estrategia prescrita se debe seguir, pero admite que hay algún margen en el orden en que los pasos pueden ser tomados, donde volver hacia atrás es a menudo necesario.

Los principales referentes epistemológicos y psicopedagógicos, así como las perspectivas y/o estrategias didácticas que se identifican en esta revisión de literatura y que juegan un papel relevante en la implementación de actividades de MSC en la enseñanza de la física, se presentan de manera sintetizada en la tabla 4.

Como ya fue mencionado, en parte de los estudios revisados los elementos epistemológicos, psicopedagógicos y didácticos no necesariamente se hacen explícitos, lo que sólo nos permite (en algunos casos) hacer deducciones para lograr así comprender las articulaciones que se dan entre ellos y, el sentido que adquieren en la implementación de actividades de Modelación y Simulación Computacional. Es claro que esta es una dificultad previsible en las revisiones de literatura; sin embargo, denota la importancia de sugerir en los estudios que se realicen en esta temática una mayor articulación entre la teoría y la práctica. 


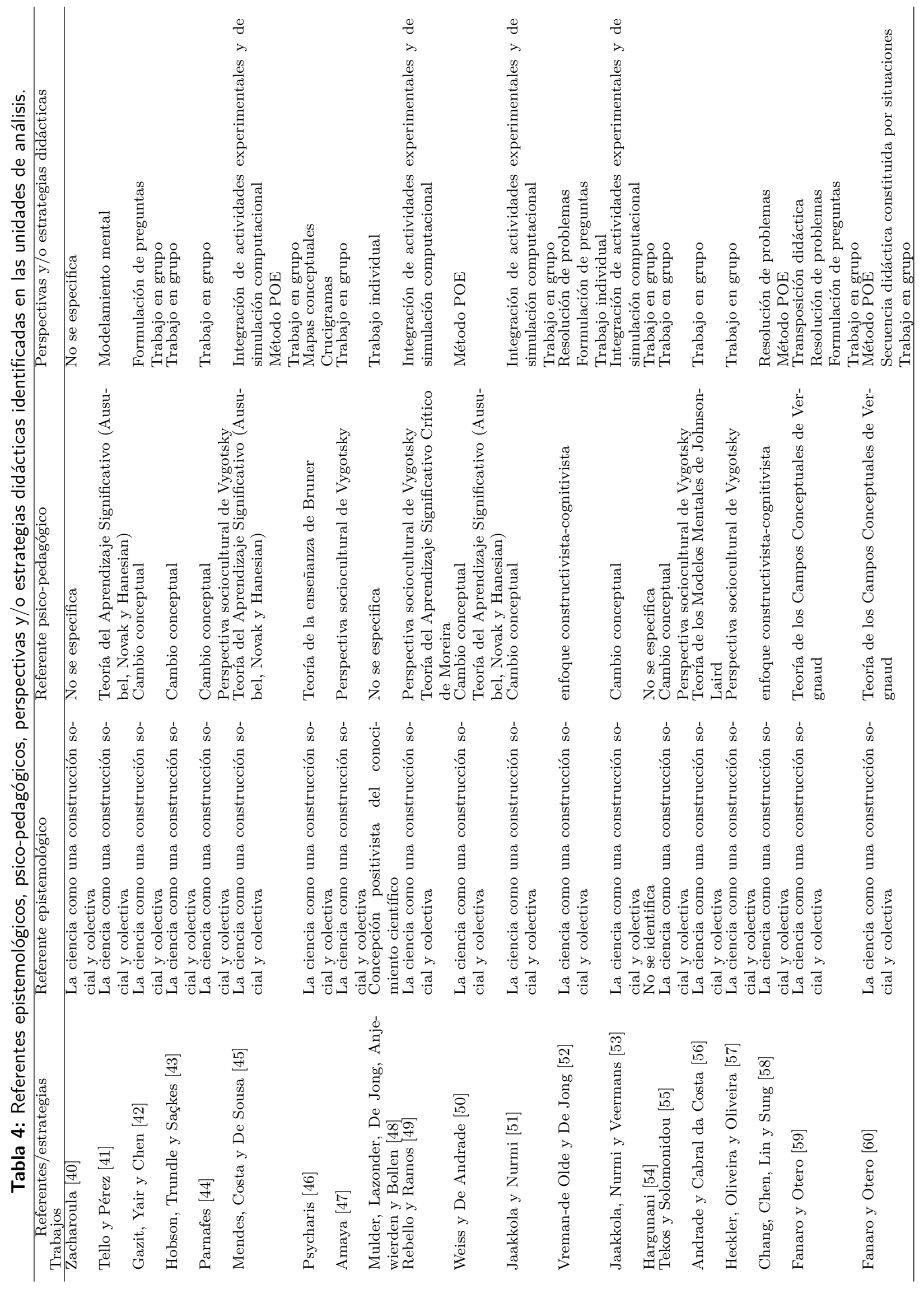




\section{Consideraciones finales}

Uno de los resultados que devela esta revisión de literatura es que la mayoría de trabajos publicados se ha concentrado esencialmente en el nivel universitario; por lo que sería importante explorar con mayor profundidad el caso de la educación básica y media; e identificar si la escasez de trabajos en este campo puede deberse a factores como la carencia de recursos computacionales para ser utilizados en el aula, las posibilidades reales que brindan las Instituciones Educativas para el uso efectivo de los mismos, la falta de formación de profesores en el uso de TIC, o el hecho de que los profesores de la educación básica y media no acostumbran hacer investigación en el aula no solo por falta de formación e interés, sino inclusive por falta de tiempo.

Teniendo en consideración que este estudio buscaba indagar, entre otras cosas, por una concepción de ciencia subyacente a la implementación de actividades de MSC, o por lo menos una concepción de modelo que se pusiera en juego en el abordaje de este tipo de actividades, encontramos que difícilmente esta concepción aparece expresada de modo explícito; y que en gran medida es vislumbrada a partir de los fundamentos psico-pedagógicos en que se sustentan los trabajos y de las perspectivas y/o estrategias didácticas utilizadas para orientar el uso de la MSC en el aula de clase; así como de la coherencia entre ellos.

En este sentido, valoramos ampliamente el hecho de que la investigación sobre la implementación de actividades de Modelación y Simulación Computacional en las clases de física en general esté fundamentada en referentes psico-pedagógicos altamente pertinentes para intentar dar cuenta de los procesos cognitivos que se generan en los estudiantes al interactuar con estas actividades. Solamente basados en fundamentos teórico-metodológicos sólidos y consistentes, con el acompañamiento de todo el proceso de desarrollo de actividades de MSC, podrán lograrse avances significativos en el campo de investigación sobre la contribución de las TIC para el aprendizaje de conocimientos de física. Pues muchas de las limitaciones que existen hoy en día a la hora de utilizar las computadoras en la enseñanza se deben, en gran medida, a la teoría psico-pedagógica en que se sustenta, o peor aún a la carencia de ésta.

De otro lado, sorprende que pese al auge que han tenido los modelos y las simulaciones computacionales en la enseñanza de las ciencias en las últimas dos décadas [20, 73 a 76], el número de trabajos que realizan una implementación efectiva de actividades de Modelación y/o Simulación Computacional en la enseñanza de la física para los niveles de educación básica y media, siga siendo aún escaso. No obstante, encontramos bastante favorecedor la existencia en los trabajos revisados de una amplia variedad de estrategias didácticas utilizadas por los docentes e investigadores en esta área para orientar los procesos de Modelación y Simulación Computacional al interior del aula de clase, propendiendo por espacios de construcción y apropiación de significados a partir del uso de estas actividades; y superando el uso meramente instrumental de los modelos y/o simulaciones, donde predominan las instrucciones precisas y la automatización de procesos.

Un elemento importante de resaltar a partir de esta revisión de literatura se refiere a la baja utilización de actividades de modelación computacional, que seguramente es producto de la complejidad que en muchos casos implica el abordaje de éstas, por razones previamente mencionadas. No obstante, advertimos que en las simulaciones computacionales previamente diseñadas, el profesor puede encontrar otra alternativa para la enseñanza de conceptos científicos que se caracterizan por un alto grado de abstracción, lo cual propiciaría otros modos de representación y de lenguaje. Esto nos lleva a considerar que la inclusión de Tecnologías de la Información y la Comunicación para la enseñanza de las ciencias en general remite hoy, no a la novedad de unos aparatos, sino a nuevos modos de percepción y de lenguaje [77]. Y es que el lenguaje mismo se resignifica a partir de la tecnología, de tal manera que el lenguaje verbal y escrito es complementado hoy por el lenguaje iconográfico, la imagen digital y los variados sistemas de representación que traen consigo nuevas maneras de pensamiento visual [78].

Finalmente, consideramos que un acercamiento a conocer el estado actual de la investigación en el campo de la Modelación y Simulación Computacional en la enseñanza de la física se constituye en una valiosa oportunidad para llevar a cabo importantes reflexiones sobre la manera como se han venido implementando estas modalidades de uso del computador en los procesos de enseñanza y aprendizaje de conceptos físicos en la educación básica y media. Del mismo modo, este conocimiento devela un panorama en la formulación de nuevas propuestas para la enseñanza de la física, en términos de la generación de aportes psico-pedagógicos, epistemológicos y didácticos para la formación de profesores de esta área, que en la actualidad no pueden ser ajenos a 
las posibilidades que brindan las TIC como recursos didácticos en los procesos educativos.

\section{Agradecimientos}

Agradecemos al Comité para el Desarrollo de la Investigación (CODI) de la Universidad de Antioquia y su convocatoria programática Ciencias Sociales, Humanidades y Artes 2012, por la financiación total del proyecto de investigación que da lugar a este producto.

\section{Referencias}

[1] M. González, V. Capuano y J. Zalazar, in: Memorias de REF XVI, organizada por APFA y las Facultades de Ingeniería y de Filosofía, Humanidades y Artes, de la U.N. de San Juan, 12 p (2009).

[2] M.A. Moreira, Revista Chilena de Educación Científica 3, 10 (2003).

[3] A.F. Cachapuz, B. Lopes, F. Paixão, J.F. Praia y C. Guerra, Revista Eureka sobre Enseñanza y Divulgación de las Ciencias 3, 167 (2006).

[4] V. Capuano, Revista Virtualidad, Educacion y Ciencia 2, 79 (2011).

[5] J. Osborne and S. Hennessy, Literature Review of ICT: Promise, Problems and Future Directions (Futurelab, Bristol, 2003).

[6] M.A. Agudelo, Revista ieRed: Revista Electrónica de la Red de Investigación Educativa [en línea] 1, 1 (2004).

[7] D. Gil, J. Carrascosa y F. Martínez, Revista Educación y Pedagogía 11, 13 (1999).

[8] M.P. Vidal, Revista Latinoamericana de Tecnología Educativa 5, 539 (2006).

[9] A. Garritz, Enseñanza de las Ciencias 28, 315 (2010).

[10] S. Hennessy, B. Onguko, D. Harrison, E.K. Ang'ondi, S. Namalefe, A. Naseem and L. Wamakote, Developing the Use of Information and Communication Technology to Enhance Teaching and Learning in East African Schools: Review of the Literature (University of Cambridge, Cambridge, 2010).

[11] M. Claro, Impacto de las tic en los Aprendizajes de los Estudiantes: Estado del Arte (Comisión Económica para América Latina y el Caribe, Naciones Unidas, 2010).

[12] M. Nussbaum y P. Rodríguez, en: Conferencia Internacional Impacto de las tic en Educación (2010), disponible en http://licroelrodas.info/ admin/Insercion/actividades/a02c5d_TICS\% 20EN\%20EDUCACION . pdf .

[13] J.C. Méndez, Revista Virtual Universidad Católica del Norte 34, 35 (2011).
[14] A. Vacchieri, Programa tic y Educación Básica: Estado del Arte Sobre la Gestión de las Políticas de Integración de Computadoras y Dispositivos Móviles en los Sistemas Educativos (Unicef, Nueva York, 2013).

[15] R.E. Lizárraga, Etic@ net 1, 1 (2014).

[16] O. Hernández, H. Jurado y Y. Romero, Revista Colombiana de Educación 66, 103 (2014).

[17] C. Murphy, Literature Review in Primary Science and ICT (Futurelab, Bristol, 2003).

[18] K. Scalise, M. Timms, A. Moorjani, L. Clark, K. Holtermann and P.S. Irvin, Journal of Research in Science Teaching 48, 1050 (2011).

[19] L.K. Smetana and R.L. Bell, International Journal of Science Education 34, 1337 (2012).

[20] I.S. Araujo, E.A. Veit e M.A. Moreira, Revista Brasileira de Ensino de Física 26, 179 (2004).

[21] I. Lawrence, in: GIREP Conference, Amsterdam (2006).

[22] L. Rogers, in: GIREP Conference, Amsterdam (2006).

[23] I.S. Araujo, E.A. Veit y M.A. Moreira, Revista Electrónica de Enseñanza de las Ciencias 6, 601 (2007).

[24] M. Bunge, Teoría y Realidad (Ariel, Barcelona, 1972).

[25] R. Giere, Explaining Science. A Cognitive Approach (University of Chicago Press, Chicago, 1988).

[26] D. Hestenes, in: Thinking Physics for Teaching, edited by C. Bernardini, C. Tarsitani and M. Vincentini (Editora Plenum Press, New York, 1995), p. 25-66.

[27] I. Halloun, Journal of Research in Science Teaching 33, 1019 (1996).

[28] I. Halloun, Modeling Theory in Science Education (Kluwer Academic Publishers Dordrecht, Boston, 2004).

[29] R.V. Brandão, I.S. Araujo, E.A. Veit y F. Lang da Silveira, Revista Electrónica de Investigación en Educación en Ciencias 6, 43 (2011).

[30] R.V. Brandão, Modelagem Científica no Ensino de Física: Conceitualização, Progressividade e Domínio por Parte de Professores em Formação Continuada. Exame de Qualificação ao Doutorado, UFRGS, Porto Alegre, 2011, 199 p.

[31] A. Adúriz-Bravo, M. Izquierdo y A. Estany, Enseñanza de las Ciencias, 20, 465 (2002).

[32] A. Bertelle, C. Iturralde y A. Rocha, Revista Iberoamericana de Educación 37, 1 (2006).

[33] J. Acevedo, A. Vázquez, M. Manassero y P. Acevedo, Revista Eureka sobre Enseñanza y Divulgación de las Ciencias 4, 202 (2007).

[34] G. Aldana, Educación y Educadores 11, 61 (2008).

[35] E. Ravanal y M. Quintanilla, Revista Electrónica de Enseñanza de las Ciencias 9, 111 (2010).

[36] G. Santos, M.R. Otero y M.A. Fanaro, Caderno Catarinense de Ensino de Física 17, 50 (2000). 
[37] A. Medeiros y C.F. Medeiros, Revista Brasileira de Ensino de Física 24, 77 (2002).

[38] C. Hoyos, Un Modelo para Investigación Documental: Guía Teórico-Práctica Sobre Construcción de Estados del Arte con Importantes Reflexiones Sobre la Investigación (Señal Editora, Medellín, 2000).

[39] J.L. Piñuel, Sociolinguistic Studies 3, 1 (2002).

[40] S. Zacharoula, Journal of Science Education 8, 99 (2007).

[41] J. Tello y C. Pérez, Revista Chilena de Educación Científica 6, 43 (2008).

[42] E. Gazit, Y. Yair y D. Chen, Journal of Sience Education and Technology 14, 459 (2005).

[43] S. Hobson, K. Trundle y M. Saçkes, Journal of Science Education and Technology 19, 165 (2010).

[44] O. Parnafes, Journal of Science Education and Technology 19, 565 (2010).

[45] J.F. Mendes, I.F. Costa y C. De Sousa, Revista Brasileira de Ensino de Física 34, 2402 (2012).

[46] S. Psycharis, Journal of Science Education 8, 38 (2007).

[47] G. Amaya, Tecné, Episteme y Didaxis: TED 25, 62 (2009).

[48] Y. Mulder, A. Lazonder, T. De Jong, A. Anjewierden y L. Bollen, Journal of Science Education and Technology 21, 722 (2012).

[49] A. Rebello y M. Ramos, Revista Experiências em Ensino de Ciências 4, 23 (2009).

[50] J. Weiss y A. De Andrade, Revista Experiências em Ensino de Ciências 1, 43 (2006).

[51] T. Jaakkola and S. Nurmi, Journal of Computer Assisted Learning 24, 271 (2008).

[52] C. Vreman-de Olde and T. De Jong, International Journal of Science Education 26, 859 (2004).

[53] T. Jaakkola, S. Nurmi and K. Veermans, Journal of Research in Science Teaching 48, 71 (2011).

[54] S. Hargunani, Latin American Journal of Physics Education 4, 520 (2010).

[55] G. Tekos and C. Solomonidou, Journal of Science Education and Technology 18, 415 (2009).

[56] M. Andrade y S. Cabral da Costa, Revista Experiências em Ensino de Ciências 1, 18 (2006).

[57] V. Heckler, M.F. Oliveira e K. Oliveira, Revista Brasileira de Ensino de Física 29, 267 (2007).

[58] K. Chang, Y. Chen, H. Lin and Y. Sung, Computers \& Education 51, 1486 (2008).

[59] M.A. Fanaro y M.R. Otero, Revista de Investigación 73, 85 (2011).

[60] M.A. Fanaro y M.R. Otero, Latin American Journal of Physics Education 2, 103 (2008).

[61] I. Fernández, D. Gil, J. Carrascosa, A. Cachapuz y J. Praia, Enseñanza de las Ciencias 20, 477 (2002).

[62] V.Y. Mellado y D. Carracedo, Enseñanza de las Ciencias 11, 331 (1993).

[63] D.P. Ausubel, J.D. Novak y H. Hanesian, Psicología Educativa: Un Punto de Vista Cogniscitivo (Editorial Trillas, México, 1983).
[64] S. Vosniadou and W.F. Brewer, Cognitive Science 18, 123 (1994).

[65] M. Limon y L. Mason, Reconsidering Conceptual Change: Issues in Theory and Practice (Kluwer, Dordrecht, 2002)

[66] I. Greca e M.A. Moreira, Revista Ciência \& Educação 9, 301 (2003).

[67] M.A. Moreira, Aprendizaje Significativo Crítico (UFRGS, Porto alegre, 2005.

[68] P.K. Tao and R.F. Gunstone, Journal of Research in Science Teaching 36, 859 (1999).

[69] Y. Chevallard, Recherchesen Didactique des Mathématiques 12, 73 (1992).

[70] M. Freschi y M. Ramos, Revista Electrónica de Enseñanza de las Ciencias 8, 156 (2009).

[71] D. Hestenes, American Journal Physics 55, 440 (1987).

[72] D. Hestenes y colaboradores, Modeling Theory of Physics Instruction, focus of educational research; Implemented through Modeling Instruction Program, in Arizona State University from 1985 to 2005 (2005).

[73] G. Camiletti e L. Ferracioli, Revista Brasileira de Ensino de Física 24, 110 (2002).

[74] C.E. Aguiar e G. Rubini, Revista Brasileira de Ensino de Física 26, 297 (2004).

[75] R.N. Giere, Philosophical Studies 143, 69 (2009).

[76] S. López, E.A. Veit y I.S. Araujo, Revista Electrónica de Enseñanza de las Ciencias 10, 202 (2011).

[77] J. Martín-Barbero, Revista Nómadas 5 (1996).

[78] J. Duarte, Estudios Pedagógicos 29, 97 (2003). 